Kragujevac Journal of Mathematics

Volume 45(3) (2021), Pages 379-392.

\title{
APPLICATIONS OF FRACTIONAL DERIVATIVE ON A DIFFERENTIAL SUBORDINATIONS AND SUPERORDINATORS FOR ANALYTIC FUNCTIONS ASSOCIATED WITH DIFFERENTIAL OPERATOR
}

\author{
ABBAS KAREEM WANAS ${ }^{1}$ AND MASLINA DARUS ${ }^{2}$
}

\begin{abstract}
The purpose of this paper is to derive subordination and superordination results involving fractional derivative of differential operator for analytic functions in the open unit disk. These results are applied to obtain sandwich results. Our results extend corresponding previously known results.
\end{abstract}

\section{Introduction and Preliminaries}

Let $\mathcal{H}=\mathcal{H}(U)$ denote the class of analytic functions in the open unit disk $U=$ $\{z \in C:|z|<1\}$. For $a \in \mathbb{C}$ and $n \in \mathbb{N}$, let $\mathcal{H}[a, n]$ be the subclass of $\mathcal{H}$ consisting of functions of the form:

$$
f(z)=a+a_{n} z^{n}+a_{n+1} z^{n+1}+\cdots, \quad a \in \mathbb{C} .
$$

Also, let $A$ be the subclass of $\mathcal{H}$ consisting of functions of the form:

$$
f(z)=z+\sum_{k=2}^{\infty} a_{k} z^{k}
$$

Let $f, g \in \mathcal{H}$. The function $f$ is said to be subordinate to $g$, or $g$ is said to be superordinate to $f$, if there exists a Schwarz function $w$ analytic in $U$ with $w(0)=0$ and $|w(z)|<1, z \in U$, such that $f(z)=g(w(z))$. This subordination is denoted by $f \prec g$ or $f(z) \prec g(z), z \in U$. It is well known that, if the function $g$ is univalent in $U$, then $f \prec g$ if and only if $f(0)=g(0)$ and $f(U) \subset g(U)$. Let $p, h \in \mathcal{H}$ and

Key words and phrases. Analytic functions, differential subordination, differential superordination, fractional derivative, differential operator.

2010 Mathematics Subject Classification. Primary: 30C45. Secondary: 30A20.

DOI 10.46793/KgJMat2103.379W

Received: August 10, 2018.

Accepted: January 23, 2019. 
$\psi(r, s, t ; z): \mathbb{C}^{3} \times U \rightarrow \mathbb{C}$. If $p$ and $\psi\left(p(z), z p^{\prime}(z), z^{2} p^{\prime \prime}(z) ; z\right)$ are univalent functions in $U$ and if $p$ satisfies the second-order differential superordination

$$
h(z) \prec \psi\left(p(z), z p^{\prime}(z), z^{2} p^{\prime \prime}(z) ; z\right),
$$

then $p$ is called a solution of the differential superordination (1.2). An analytic function $q$ is called a subordinate of (1.2), if $q \prec p$ for all $p$ satisfying (1.2). An univalent subordinat $\tilde{q}$ that satisfies $q \prec \tilde{q}$ for all the subordinants $q$ of (1.2) is called the best subordinant.

Miller and Mocanu [6] obtained conditions on the functions $h, q$ and $\psi$ for which the following implication holds:

$$
h(z) \prec \psi\left(p(z), z p^{\prime}(z), z^{2} p^{\prime \prime}(z) ; z\right) \Rightarrow q(z) \prec p(z) .
$$

Ali et al. [1] have used the results of Bulboacǎ [3] to obtain sufficient conditions for certain normalized analytic functions to satisfy

$$
q_{1}(z) \prec \frac{z f^{\prime}(z)}{f(z)} \prec q_{2}(z),
$$

where $q_{1}$ and $q_{2}$ are given univalent functions in $U$ with $q_{1}(0)=q_{2}(0)=1$.

Also, Tuneski [16] obtain sufficient condition for starlikeness of $f \in A$ in terms of the quantity $\frac{f^{\prime \prime}(z) f(z)}{\left(f^{\prime}(z)\right)^{2}}$. Shanmugam et al. [14], Goyal et al. [4], Wanas [17,18] and Attiya and Yassen [2] have obtained sandwich results for certain classes of analytic functions.

Definition $1.1([9])$. For $f \in A$ the operator $I_{\lambda_{1}, \lambda_{2}, \ell, d}^{n, m}$ is defined by $I_{\lambda_{1}, \lambda_{2}, \ell, d}^{n, m}: A \rightarrow A$,

$$
I_{\lambda_{1}, \lambda_{2}, \ell, d}^{n, m} f(z)=\mathcal{M}_{\lambda_{1}, \lambda_{2}, \ell, d}^{m}(z) * R^{n} f(z), \quad z \in U,
$$

where

$$
\mathcal{M}_{\lambda_{1}, \lambda_{2}, \ell, d}^{m}(z)=z+\sum_{k=2}^{\infty}\left[\frac{\ell\left(1+\left(\lambda_{1}+\lambda_{2}\right)(k-1)\right)+d}{\ell\left(1+\lambda_{2}(k-1)\right)+d}\right]^{m} z^{k},
$$

and $R^{n} f(z)$ denotes the Ruscheweyh derivative operator [10] given by

$$
R^{n} f(z)=z+\sum_{k=2}^{\infty} C(n, k) a_{k} z^{k}
$$

where $C(n, k)=\frac{\Gamma(k+n)}{\Gamma(n+1) \Gamma(k)}, n, m \in \mathbb{N}_{0}=\mathbb{N} \cup\{0\}, \lambda_{2} \geq \lambda_{1} \geq 0, \ell \geq 0$ and $\ell+d>0$.

If $f$ given by (1.1), then we easily find that

$$
I_{\lambda_{1}, \lambda_{2}, \ell, d}^{n, m} f(z)=z+\sum_{k=2}^{\infty} \frac{\Gamma(k+n)}{\Gamma(n+1) \Gamma(k)}\left[\frac{\ell\left(1+\left(\lambda_{1}+\lambda_{2}\right)(k-1)\right)+d}{\ell\left(1+\lambda_{2}(k-1)\right)+d}\right]^{m} a_{k} z^{k}
$$

Definition $1.2([15])$. The fractional derivative of order $\delta, 0 \leq \delta<1$, of a function $f$ is defined by

$$
D_{z}^{\delta} f(z)=\frac{1}{\Gamma(1-\delta)} \frac{d}{d z} \int_{0}^{z} \frac{f(t)}{(z-t)^{\delta}} d t
$$


where the function $f$ is analytic in a simply-connected region of the z-plane containing the origin and the multiplicity of $(z-t)^{-\delta}$ is removed by requiring $\log (z-t)$ to be real, when $\operatorname{Re}(z-t)>0$.

From Definition 1.1 and Definition 1.2, we have

$$
\begin{aligned}
D_{z}^{\delta} I_{\lambda_{1}, \lambda_{2}, \ell, d}^{n, m} f(z)= & \frac{1}{\Gamma(2-\delta)} z^{1-\delta}+\sum_{k=2}^{\infty} \frac{k \Gamma(n+k)}{\Gamma(k-\delta+1) \Gamma(n+1)} \\
& \times\left[\frac{\ell\left(1+\left(\lambda_{1}+\lambda_{2}\right)(k-1)\right)+d}{\ell\left(1+\lambda_{2}(k-1)\right)+d}\right]^{m} a_{k} z^{k-\delta} .
\end{aligned}
$$

It follows from (1.3) that

$$
\begin{aligned}
\ell \lambda_{1} z\left(D_{z}^{\delta} I_{\lambda_{1}, \lambda_{2}, \ell, d}^{n, m} f(z)\right)^{\prime}= & {\left[\ell\left(1+\left(\lambda_{2}(k-1)\right)+d\right] D_{z}^{\delta} I_{\lambda_{1}, \lambda_{2}, \ell, d}^{n, m+1} f(z)\right.} \\
& -\left[\ell\left(1+\left(\lambda_{2}(k-1)-(1-\delta) \lambda_{1}\right)+d\right] D_{z}^{\delta} I_{\lambda_{1}, \lambda_{2}, \ell, d}^{n, m} f(z) .\right.
\end{aligned}
$$

In order to prove our results, we make use of the following known results.

Definition $1.3([5])$. Denote by $Q$ the set of all functions $f$ that are analytic and injective on $\bar{U} \backslash E(f)$, where

$$
E(f)=\left\{\zeta \in \partial U: \lim _{z \rightarrow \zeta} f(z)=\infty\right\}
$$

and are such that $f^{\prime}(\zeta) \neq 0$ for $\zeta \in \partial U \backslash E(f)$.

Lemma $1.1([5])$. Let $q$ be univalent in the unit disk $U$ and let $\theta$ and $\phi$ be analytic in a domain $D$ containing $q(U)$, with $\phi(w) \neq 0$ when $w \in q(U)$. Set $Q(z)=z q^{\prime}(z) \phi(q(z))$ and $h(z)=\theta(q(z))+Q(z)$. Suppose that

(1) $Q(z)$ is starlike univalent in $U$;

(2) $\operatorname{Re}\left\{\frac{z h^{\prime}(z)}{Q(z)}\right\}>0$ for $z \in U$.

If $p$ is analytic in $U$, with $p(0)=q(0), p(U) \subset D$ and

$$
\theta(p(z))+z p^{\prime}(z) \phi(p(z)) \prec \theta(q(z))+z q^{\prime}(z) \phi(q(z)),
$$

then $p \prec q$ and $q$ is the best dominant of (1.5).

Lemma $1.2([6])$. Let $q$ be a convex univalent function in $U$ and let $\alpha \in C, \beta \in C \backslash\{0\}$, with

If $p$ is analytic in $U$ and

$$
\operatorname{Re}\left\{1+\frac{z q^{\prime \prime}(z)}{q^{\prime}(z)}\right\}>\max \left\{0,-\operatorname{Re}\left(\frac{\alpha}{\beta}\right)\right\} .
$$

$$
\alpha p(z)+\beta z p^{\prime}(z) \prec \alpha q(z)+\beta z q^{\prime}(z),
$$

then $p \prec q$ and $q$ is the best dominant of (1.6). 
Lemma $1.3([6])$. Let $q$ be a convex univalent function in $U$ and let $\beta \in \mathbb{C}$. Further assume that $\operatorname{Re}(\beta)>0$. If $p \in \mathcal{H}[q(0), 1] \cap Q$ and $p(z)+\beta z p^{\prime}(z)$ is univalent in $U$, then

$$
q(z)+\beta z q^{\prime}(z) \prec p(z)+\beta z p^{\prime}(z)
$$

which implies that $q \prec p$ and $q$ is the best subordinant of (1.7).

Lemma $1.4([3])$. Let $q$ be convex univalent in the unit disk $U$ and let $\theta$ and $\phi$ be analytic in a domain $D$ containing $q(U)$. Suppose that

(1) $\operatorname{Re}\left\{\frac{\theta^{\prime}(q(z))}{\phi(q(z))}\right\}>0$ for $z \in U$;

(2) $Q(z)=z q^{\prime}(z) \phi(q(z))$ is starlike univalent in $U$.

If $p \in \mathcal{H}[q(0), 1] \cap Q$, with $p(U) \subset D, \theta(p(z))+z p^{\prime}(z) \phi(p(z))$ is univalent in $U$, and

$$
\theta(q(z))+z q^{\prime}(z) \phi(q(z)) \prec \theta(p(z))+z p^{\prime}(z) \phi(p(z)),
$$

then $q \prec p$ and $q$ is the best subordinant of (1.8).

\section{Subordination Results}

Theorem 2.1. Let $q$ be convex univalent in $U$ with $q(0)=1, \sigma \in \mathbb{C} \backslash\{0\}, \gamma>0$ and suppose that $q$ satisfies

$$
\operatorname{Re}\left\{1+\frac{z q^{\prime \prime}(z)}{q^{\prime}(z)}\right\}>\max \left\{0,-\operatorname{Re}\left(\frac{(1-\delta) \gamma}{\sigma}\right)\right\} .
$$

If $f \in A$ satisfies the subordination

$$
\begin{aligned}
& \left(1-\frac{\sigma\left[\ell\left(1+\left(\lambda_{2}(k-1)\right)+d\right]\right.}{\ell \lambda_{1}(1-\delta)}\right)\left(\frac{\Gamma(2-\delta) D_{z}^{\delta} I_{\lambda_{1}, \lambda_{2}, \ell, d}^{n, m} f(z)}{z^{1-\delta}}\right)^{\gamma} \\
+ & \frac{\sigma\left[\ell\left(1+\left(\lambda_{2}(k-1)\right)+d\right]\right.}{\ell \lambda_{1}(1-\delta)}\left(\frac{\Gamma(2-\delta) D_{z}^{\delta} I_{\lambda_{1}, \lambda_{2}, \ell, d}^{n, m} f(z)}{z^{1-\delta}}\right)^{\gamma}\left(\frac{D_{z}^{\delta} I_{\lambda_{1}, \lambda_{2}, \ell, d}^{n, m+1} f(z)}{D_{z}^{\delta} I_{\lambda_{1}, \lambda_{2}, \ell, d}^{n, m} f(z)}\right) \\
\prec & q(z)+\frac{\sigma}{(1-\delta) \gamma} z q^{\prime}(z),
\end{aligned}
$$

then

$$
\left(\frac{\Gamma(2-\delta) D_{z}^{\delta} I_{\lambda_{1}, \lambda_{2}, \ell, d}^{n, m} f(z)}{z^{1-\delta}}\right)^{\gamma} \prec q(z)
$$

and $q$ is the best dominant of (2.2).

Proof. Define the function $p$ by

$$
p(z)=\left(\frac{\Gamma(2-\delta) D_{z}^{\delta} I_{\lambda_{1}, \lambda_{2}, \ell, d}^{n, m} f(z)}{z^{1-\delta}}\right)^{\gamma}, \quad z \in U .
$$


Then the function $p$ is analytic in $U$ and $p(0)=1$. Differentiating (2.4) logarithmically with respect to $z$, we have

$$
\frac{z p^{\prime}(z)}{p(z)}=\gamma\left(\frac{z\left(D_{z}^{\delta} I_{\lambda_{1}, \lambda_{2}, \ell, d}^{n, m} f(z)\right)^{\prime}}{D_{z}^{\delta} I_{\lambda_{1}, \lambda_{2}, \ell, d}^{n, d} f(z)}-(1-\delta)\right) .
$$

Now, in view of (1.4), we obtain

$$
\frac{z p^{\prime}(z)}{p(z)}=\frac{\gamma\left[\ell\left(1+\left(\lambda_{2}(k-1)\right)+d\right]\right.}{\ell \lambda_{1}}\left(\frac{D_{z}^{\delta} I_{\lambda_{1}, \lambda_{2}, \ell, d}^{n, m+1} f(z)}{D_{z}^{\delta} I_{\lambda_{1}, \lambda_{2}, \ell, d}^{n, m} f(z)}-1\right) .
$$

Therefore,

$$
\begin{aligned}
\frac{z p^{\prime}(z)}{(1-\delta) \gamma}= & \frac{\ell\left(1+\left(\lambda_{2}(k-1)\right)+d\right.}{\ell \lambda_{1}(1-\delta)}\left(\frac{\Gamma(2-\delta) D_{z}^{\delta} I_{\lambda_{1}, \lambda_{2}, \ell, d}^{n, m} f(z)}{z^{1-\delta}}\right)^{\gamma} \\
& \times\left(\frac{D_{z}^{\delta} I_{\lambda_{1}, \lambda_{2}, \ell, d}^{n+m+1} f(z)}{D_{z}^{\delta} I_{\lambda_{1}, \lambda_{2}, \ell, d}^{n, m} f(z)}-1\right) .
\end{aligned}
$$

It follows from (2.2) that

$$
p(z)+\frac{\sigma}{(1-\delta) \gamma} z p^{\prime}(z) \prec q(z)+\frac{\sigma}{(1-\delta) \gamma} z q^{\prime}(z) .
$$

Thus, an application of Lemma 1.2, with $\alpha=1$ and $\beta=\frac{\sigma}{(1-\delta) \gamma}$, we obtain (2.3).

Theorem 2.2. Let $\eta_{i} \in \mathbb{C}, i=1,2,3,4, \gamma>0, t \in \mathbb{C} \backslash\{0\}$ and $q$ be convex univalent in $U$ with $q(0)=1, q(z) \neq 0, z \in U$, and assume that $q$ satisfies

$$
\operatorname{Re}\left\{1+\frac{\eta_{2}}{t} q(z)+\frac{2 \eta_{3}}{t} q^{2}(z)+\frac{3 \eta_{4}}{t} q^{3}(z)+\frac{z q^{\prime \prime}(z)}{q^{\prime}(z)}-\frac{z q^{\prime}(z)}{q(z)}\right\}>0 .
$$

Suppose that $\frac{z q^{\prime}(z)}{q(z)}$ is starlike univalent in $U$. If $f \in A$ satisfies

$\Psi\left(\eta_{1}, \eta_{2}, \eta_{3}, \eta_{4}, \gamma, t, \delta, n, m, \lambda_{1}, \lambda_{2}, \ell, d ; z\right) \prec \eta_{1}+\eta_{2} q(z)+\eta_{3} q^{2}(z)+\eta_{4} q^{3}(z)+t \frac{z q^{\prime}(z)}{q(z)}$ where

$$
\begin{aligned}
& \Psi\left(\eta_{1}, \eta_{2}, \eta_{3}, \eta_{4}, \gamma, t, \delta, n, m, \lambda_{1}, \lambda_{2}, \ell, d ; z\right) \\
= & \eta_{1}+\eta_{2}\left(\frac{D_{z}^{\delta} I_{\lambda_{1}, \lambda_{2}, \ell, d}^{n, m+1} f(z)}{D_{z}^{\delta} I_{\lambda_{1}, \lambda_{2}, \ell, d}^{n, m} f(z)}\right)^{\gamma}+\eta_{3}\left(\frac{D_{z}^{\delta} I_{\lambda_{1}, \lambda_{2}, \ell, d}^{n, m+1} f(z)}{D_{z}^{\delta} I_{\lambda_{1}, \lambda_{2}, \ell, d}^{n, m} f(z)}\right)^{2 \gamma}+\eta_{4}\left(\frac{D_{z}^{\delta} I_{\lambda_{1}, \lambda_{2}, \ell, d}^{n, m} f(z)}{D_{z}^{\delta} I_{\lambda_{1}, \lambda_{2}, \ell, d}^{n, m} f(z)}\right)^{3 \gamma} \\
& +\frac{\gamma t\left[\ell\left(1+\left(\lambda_{2}(k-1)\right)+d\right]\right.}{\ell \lambda_{1}}\left(\frac{D_{z}^{\delta} I_{\lambda_{1}, \lambda_{2}, \ell, d}^{n, m+2} f(z)}{D_{z}^{\delta} I_{\lambda_{1}, \lambda_{2}, \ell, d}^{n, m+1} f(z)}-\frac{D_{z}^{\delta} I_{\lambda_{1}, \lambda_{2}, \ell, d}^{n, m+1} f(z)}{D_{z}^{\delta} I_{\lambda_{1}, \lambda_{2}, \ell, d}^{n, m} f(z)}\right)
\end{aligned}
$$


then

$$
\left(\frac{D_{z}^{\delta} I_{\lambda_{1}, \lambda_{2}, \ell, d}^{n, m+1} f(z)}{D_{z}^{\delta} I_{\lambda_{1}, \lambda_{2}, \ell, d}^{n, m} f(z)}\right)^{\gamma} \prec q(z)
$$

and $q$ is the best dominant of (2.6).

Proof. Define the function $p$ by

$$
p(z)=\left(\frac{D_{z}^{\delta} I_{\lambda_{1}, \lambda_{2}, \ell, d}^{n, m+1} f(z)}{D_{z}^{\delta} I_{\lambda_{1}, \lambda_{2}, \ell, d}^{n, m} f(z)}\right)^{\gamma}, \quad z \in U .
$$

Then the function $p$ is analytic in $U$ and $p(0)=1$.

By a straightforward computation and using (1.4), we have

$$
\eta_{1}+\eta_{2} p(z)+\eta_{3} p^{2}(z)+\eta_{4} p^{3}(z)+t \frac{z p^{\prime}(z)}{p(z)}=\Psi\left(\eta_{1}, \eta_{2}, \eta_{3}, \eta_{4}, \gamma, t, \delta, n, m, \lambda_{1}, \lambda_{2}, \ell, d ; z\right),
$$

where $\Psi\left(\eta_{1}, \eta_{2}, \eta_{3}, \eta_{4}, \gamma, t, \delta, n, m, \lambda_{1}, \lambda_{2}, \ell, d ; z\right)$ is given by (2.7). From (2.6) and (2.9), we obtain

$\eta_{1}+\eta_{2} p(z)+\eta_{3} p^{2}(z)+\eta_{4} p^{3}(z)+t \frac{z p^{\prime}(z)}{p(z)} \prec \eta_{1}+\eta_{2} q(z)+\eta_{3} q^{2}(z)+\eta_{4} q^{3}(z)+t \frac{z q^{\prime}(z)}{q(z)}$.

By setting

$$
\theta(w)=\eta_{1}+\eta_{2} w+\eta_{3} w^{2}+\eta_{4} w^{3} \quad \text { and } \quad \phi(w)=\frac{t}{w}, \quad w \neq 0,
$$

we see that $\theta(w)$ is analytic in $\mathbb{C}, \phi(w)$ is analytic in $\mathbb{C} \backslash\{0\}$ and that $\phi(w) \neq 0$, $w \in \mathbb{C} \backslash\{0\}$. Also, we get

$$
Q(z)=z q^{\prime}(z) \phi(q(z))=t \frac{z q^{\prime}(z)}{q(z)}
$$

and

$$
h(z)=\theta(q(z))+Q(z)=\eta_{1}+\eta_{2} q(z)+\eta_{3} q^{2}(z)+\eta_{4} q^{3}(z)+t \frac{z q^{\prime}(z)}{q(z)} .
$$

It is clear that $Q(z)$ is starlike univalent in $U$,

$$
\operatorname{Re}\left\{\frac{z h^{\prime}(z)}{Q(z)}\right\}=\operatorname{Re}\left\{1+\frac{\eta_{2}}{t} q(z)+\frac{2 \eta_{3}}{t} q^{2}(z)+\frac{3 \eta_{4}}{t} q^{3}(z)+\frac{z q^{\prime \prime}(z)}{q^{\prime}(z)}-\frac{z q^{\prime}(z)}{q(z)}\right\}>0 .
$$

Thus, by Lemma 1.1, we get $p(z) \prec q(z)$. By using (2.8), we obtain the desired result.

Theorem 2.3. Let $\eta_{i} \in \mathbb{C}, i=1,2,3,4, t \in V \backslash\{0\}$ and $q$ be convex univalent in $U$ with $q(0)=1, q(z) \neq 0, z \in U$, and assume that $q$ satisfies $(2.5)$. Suppose that $\frac{z q^{\prime}(z)}{q(z)}$ is starlike univalent in $U$. If $f \in A$ satisfies

$$
\Omega\left(\eta_{1}, \eta_{2}, \eta_{3}, \eta_{4}, t, \delta, n, m, \lambda_{1}, \lambda_{2}, \ell, d ; z\right) \prec \eta_{1}+\eta_{2} q(z)+\eta_{3} q^{2}(z)+\eta_{4} q^{3}(z)+t \frac{z q^{\prime}(z)}{q(z)},
$$




$$
\begin{aligned}
& \Omega\left(\eta_{1}, \eta_{2}, \eta_{3}, \eta_{4}, t, \delta, n, m, \lambda_{1}, \lambda_{2}, \ell, d ; z\right) \\
= & \eta_{1}+\eta_{2} \frac{z^{1-\delta} D_{z}^{\delta} I_{\lambda_{1}, \lambda_{2}, \ell, d}^{n, m+1} f(z)}{\Gamma(2-\delta)\left(D_{z}^{\delta} I_{\lambda_{1}, \lambda_{2}, \ell, d}^{n, m} f(z)\right)^{2}}+\eta_{3}\left(\frac{1}{\Gamma(2-\delta)}\right)^{2} \frac{z^{2(1-\delta)}\left(D_{z}^{\delta} I_{\lambda_{1}, \lambda_{2}, \ell, d}^{n, m+1} f(z)\right)^{2}}{\left(D_{z}^{\delta} I_{\lambda_{1}, \lambda_{2}, \ell, d}^{n, m} f(z)\right)^{4}} \\
& +\eta_{4}\left(\frac{1}{\Gamma(2-\delta)}\right)^{3} \frac{z^{3(1-\delta)}\left(D_{z}^{\delta} I_{\lambda_{1}, \lambda_{2}, \ell, d}^{n, m+1} f(z)\right)^{3}}{\left(D_{z}^{\delta} I_{\lambda_{1}, \lambda_{2}, \ell, d}^{n, m} f(z)\right)^{6}}+\frac{t\left[\ell\left(1+\left(\lambda_{2}(k-1)\right)+d\right]\right.}{\ell \lambda_{1}} \times \\
& \times\left(1+\frac{D_{z}^{\delta} I_{\lambda_{1}, \lambda_{2}, \ell, d}^{n, m+2} f(z)}{D_{z}^{\delta} I_{\lambda_{1}, \lambda_{2}, \ell, d}^{n, m+1} f(z)}-\frac{2 D_{z}^{\delta} I_{\lambda_{1}, \lambda_{2}, \ell, d}^{n, m+1} f(z)}{D_{z}^{\delta} I_{\lambda_{1}, \lambda_{2}, \ell, d}^{n, m} f(z)}\right),
\end{aligned}
$$

then

$$
\frac{z^{1-\delta} D_{z}^{\delta} I_{\lambda_{1}, \lambda_{2}, \ell, d}^{n, m+1} f(z)}{\Gamma(2-\delta)\left(D_{z}^{\delta} I_{\lambda_{1}, \lambda_{2}, \ell, d}^{n, m} f(z)\right)^{2}} \prec q(z)
$$

and $q$ is the best dominant of (2.10).

Proof. Define the function $p$ by

$$
p(z)=\frac{z^{1-\delta} D_{z}^{\delta} I_{\lambda_{1}, \lambda_{2}, \ell, d}^{n, m+1} f(z)}{\Gamma(2-\delta)\left(D_{z}^{\delta} I_{\lambda_{1}, \lambda_{2}, \ell, d}^{n, m} f(z)\right)^{2}}, \quad z \in U .
$$

Then the function $p$ is analytic in $U$ and $p(0)=1$.

We note that

$$
\eta_{1}+\eta_{2} p(z)+\eta_{3} p^{2}(z)+\eta_{4} p^{3}(z)+t \frac{z p^{\prime}(z)}{p(z)}=\Omega\left(\eta_{1}, \eta_{2}, \eta_{3}, \eta_{4}, t, \delta, n, m, \lambda_{1}, \lambda_{2}, \ell, d ; z\right),
$$

where $\Omega\left(\eta_{1}, \eta_{2}, \eta_{3}, \eta_{4}, t, \delta, n, m, \lambda_{1}, \lambda_{2}, \ell, d ; z\right)$ is given by (2.11). From (2.10) and (2.13), we obtain

$$
\eta_{1}+\eta_{2} p(z)+\eta_{3} p^{2}(z)+\eta_{4} p^{3}(z)+t \frac{z p^{\prime}(z)}{p(z)} \prec \eta_{1}+\eta_{2} q(z)+\eta_{3} q^{2}(z)+\eta_{4} q^{3}(z)+t \frac{z q^{\prime}(z)}{q(z)} .
$$

The remaining part of the proof Theorem 2.3 is similar to that of Theorem 2.2 and hence we omit it.

\section{Superordination Results}

Theorem 3.1. Let $q$ be convex univalent in $U$ with $q(0)=1, \gamma>0$ and $\operatorname{Re}\{\sigma\}>0$. Let $f \in A$ satisfies

$$
\left(\frac{\Gamma(2-\delta) D_{z}^{\delta} I_{\lambda_{1}, \lambda_{2}, \ell, d}^{n, m} f(z)}{z^{1-\delta}}\right)^{\gamma} \in \mathcal{H}[q(0), 1] \cap Q
$$


and

$$
\begin{aligned}
& \left(1-\frac{\sigma\left[\ell\left(1+\left(\lambda_{2}(k-1)\right)+d\right]\right.}{\ell \lambda_{1}(1-\delta)}\right)\left(\frac{\Gamma(2-\delta) D_{z}^{\delta} I_{\lambda_{1}, \lambda_{2}, \ell, d}^{n, m} f(z)}{z^{1-\delta}}\right)^{\gamma} \\
& +\frac{\sigma\left[\ell\left(1+\left(\lambda_{2}(k-1)\right)+d\right]\right.}{\ell \lambda_{1}(1-\delta)}\left(\frac{\Gamma(2-\delta) D_{z}^{\delta} I_{\lambda_{1}, \lambda_{2}, \ell, d}^{n, m} f(z)}{z^{1-\delta}}\right)^{\gamma}\left(\frac{D_{z}^{\delta} I_{\lambda_{1}, \lambda_{2}, \ell, d}^{n, m+1} f(z)}{D_{z}^{\delta} I_{\lambda_{1}, \lambda_{2}, \ell, d}^{n, m} f(z)}\right)
\end{aligned}
$$

be univalent in $U$. If

$$
\begin{aligned}
& q(z)+\frac{\sigma}{(1-\delta) \gamma} z q^{\prime}(z) \\
& \prec\left(1-\frac{\sigma\left[\ell\left(1+\left(\lambda_{2}(k-1)\right)+d\right]\right.}{\ell \lambda_{1}(1-\delta)}\right)\left(\frac{\Gamma(2-\delta) D_{z}^{\delta} I_{\lambda_{1}, \lambda_{2}, \ell, d}^{n, m} f(z)}{z^{1-\delta}}\right)^{\gamma} \\
& +\frac{\sigma\left[\ell\left(1+\left(\lambda_{2}(k-1)\right)+d\right]\right.}{\ell \lambda_{1}(1-\delta)}\left(\frac{\Gamma(2-\delta) D_{z}^{\delta} I_{\lambda_{1}, \lambda_{2}, \ell, d}^{n, m} f(z)}{z^{1-\delta}}\right)^{\gamma}\left(\frac{D_{z}^{\delta} I_{\lambda_{1}, \lambda_{2}, \ell, d}^{n, m+1} f(z)}{D_{z}^{\delta} I_{\lambda_{1}, \lambda_{2}, \ell, d}^{n, \ell} f(z)}\right)
\end{aligned}
$$

then

$$
q(z) \prec\left(\frac{\Gamma(2-\delta) D_{z}^{\delta} I_{\lambda_{1}, \lambda_{2}, \ell, d}^{n, m} f(z)}{z^{1-\delta}}\right)^{\gamma}
$$

and $q$ is the best subordinant of (3.1).

Proof. Define the function $p$ by

$$
p(z)=\left(\frac{\Gamma(2-\delta) D_{z}^{\delta} I_{\lambda_{1}, \lambda_{2}, \ell, d}^{n, m} f(z)}{z^{1-\delta}}\right)^{\gamma}, \quad z \in U .
$$

Then the function $p$ is analytic in $U$ and $p(0)=1$. Differentiating (3.3) logarithmically with respect to $z$, we get

$$
\frac{z p^{\prime}(z)}{p(z)}=\gamma\left(\frac{z\left(D_{z}^{\delta} I_{\lambda_{1}, \lambda_{2}, \ell, d}^{n, m} f(z)\right)^{\prime}}{D_{z}^{\delta} I_{\lambda_{1}, \lambda_{2}, \ell, d}^{n, d} f(z)}-(1-\delta)\right) .
$$

After some computations and using (1.4), we find that

$$
\begin{aligned}
& \left(1-\frac{\sigma\left[\ell\left(1+\left(\lambda_{2}(k-1)\right)+d\right]\right.}{\ell \lambda_{1}(1-\delta)}\right)\left(\frac{\Gamma(2-\delta) D_{z}^{\delta} I_{\lambda_{1}, \lambda_{2}, \ell, d}^{n, m} f(z)}{z^{1-\delta}}\right)^{\gamma} \\
& +\frac{\sigma\left[\ell\left(1+\left(\lambda_{2}(k-1)\right)+d\right]\right.}{\ell \lambda_{1}(1-\delta)}\left(\frac{\Gamma(2-\delta) D_{z}^{\delta} I_{\lambda_{1}, \lambda_{2}, \ell, d}^{n, m} f(z)}{z^{1-\delta}}\right)^{\gamma}\left(\frac{D_{z}^{\delta} I_{\lambda_{1}, \lambda_{2}, \ell, d}^{n, m+1} f(z)}{D_{z}^{\delta} I_{\lambda_{1}, \lambda_{2}, \ell, d}^{n, m} f(z)}\right) \\
= & p(z)+\frac{\sigma}{(1-\delta) \gamma} z p^{\prime}(z) .
\end{aligned}
$$

From (3.1) and (3.4), we have

$$
q(z)+\frac{\sigma}{(1-\delta) \gamma} z q^{\prime}(z) \prec p(z)+\frac{\sigma}{(1-\delta) \gamma} z p^{\prime}(z) .
$$


Thus, an application of Lemma 1.3, with $\alpha=1$ and $\beta=\frac{\sigma}{(1-\delta) \gamma}$, we obtain the results.

Theorem 3.2. Let $\eta_{i} \in \mathbb{C}, i=1,2,3,4, \gamma>0, t \in \mathbb{C} \backslash\{0\}$ and $q$ be convex univalent in $U$ with $q(0)=1, q(z) \neq 0, z \in U$, and assume that $q$ satisfies

$$
\operatorname{Re}\left\{\frac{\eta_{2}}{t} q(z)+\frac{2 \eta_{3}}{t} q^{2}(z)+\frac{3 \eta_{4}}{t} q^{3}(z)\right\}>0 .
$$

Suppose that $\frac{z q^{\prime}(z)}{q(z)}$ is starlike univalent in $U$. Let $f \in A$ satisfies

$$
\left(\frac{D_{z}^{\delta} I_{\lambda_{1}, \lambda_{2}, \ell, d}^{n, m+1} f(z)}{D_{z}^{\delta} I_{\lambda_{1}, \lambda_{2}, \ell, d}^{n, m} f(z)}\right)^{\gamma} \in H[q(0), 1] \cap Q
$$

and $\Psi\left(\eta_{1}, \eta_{2}, \eta_{3}, \eta_{4}, \gamma, t, \delta, n, m, \lambda_{1}, \lambda_{2}, \ell, d ; z\right) \quad$ is univalent in $U$, where $\Psi\left(\eta_{1}, \eta_{2}, \eta_{3}, \eta_{4}, \gamma, t, \delta, n, m, \lambda_{1}, \lambda_{2}, \ell, d ; z\right)$ is given by (2.7). If

$\eta_{1}+\eta_{2} q(z)+\eta_{3} q^{2}(z)+\eta_{4} q^{3}(z)+t \frac{z q^{\prime}(z)}{q(z)} \prec \Psi\left(\eta_{1}, \eta_{2}, \eta_{3}, \eta_{4}, \gamma, t, \delta, n, m, \lambda_{1}, \lambda_{2}, \ell, d ; z\right)$,

then

$$
q(z) \prec\left(\frac{D_{z}^{\delta} I_{\lambda_{1}, \lambda_{2}, \ell, d}^{n, m+1} f(z)}{D_{z}^{\delta} I_{\lambda_{1}, \lambda_{2}, \ell, d}^{n, f} f(z)}\right)^{\gamma}
$$

and $q$ is the best subordinant of (3.6).

Proof. Define the function $p$ by

$$
p(z)=\left(\frac{D_{z}^{\delta} I_{\lambda_{1}, \lambda_{2}, \ell, d}^{n, m+1} f(z)}{D_{z}^{\delta} I_{\lambda_{1}, \lambda_{2}, \ell, d}^{n, m} f(z)}\right)^{\gamma}, \quad z \in U .
$$

Then the function $p$ is analytic in $U$ and $p(0)=1$.

By some computation, we have

$\Psi\left(\eta_{1}, \eta_{2}, \eta_{3}, \eta_{4}, \gamma, t, \delta, n, m, \lambda_{1}, \lambda_{2}, \ell, d ; z\right)=\eta_{1}+\eta_{2} p(z)+\eta_{3} p^{2}(z)+\eta_{4} p^{3}(z)+t \frac{z p^{\prime}(z)}{p(z)}$,

where $\Psi\left(\eta_{1}, \eta_{2}, \eta_{3}, \eta_{4}, \gamma, t, \delta, n, m, \lambda_{1}, \lambda_{2}, \ell, d ; z\right)$ is given by (2.7). From (3.6) and (3.8), we obtain

$\eta_{1}+\eta_{2} q(z)+\eta_{3} q^{2}(z)+\eta_{4} q^{3}(z)+t \frac{z q^{\prime}(z)}{q(z)} \prec \eta_{1}+\eta_{2} p(z)+\eta_{3} p^{2}(z)+\eta_{4} p^{3}(z)+t \frac{z p^{\prime}(z)}{p(z)}$.

By setting $\theta(w)=\eta_{1}+\eta_{2} w+\eta_{3} w^{2}+\eta_{4} w^{3}$ and $\phi(w)=\frac{t}{w}, w \neq 0$, we see that $\theta(w)$ is analytic in $\mathbb{C}, \phi(w)$ is analytic in $\mathbb{C} \backslash\{0\}$ and that $\phi(w) \neq 0, w \in \mathbb{C} \backslash\{0\}$. Also, we get

$$
Q(z)=z q^{\prime}(z) \phi(q(z))=t \frac{z q^{\prime}(z)}{q(z)}
$$


It is clear that $Q(z)$ is starlike univalent in $U$,

$$
\operatorname{Re}\left\{\frac{\theta^{\prime}(q(z))}{\phi(q(z))}\right\}=\operatorname{Re}\left\{\frac{\eta_{2}}{t} q(z)+\frac{2 \eta_{3}}{t} q^{2}(z)+\frac{3 \eta_{4}}{t} q^{3}(z)\right\}>0 .
$$

Thus, by Lemma 1.4, we get $q(z) \prec p(z)$. By using (3.7), we obtain the desired result.

Theorem 3.3. Let $\eta_{i} \in \mathbb{C}, i=1,2,3,4, t \in \mathbb{C} \backslash\{0\}$ and $q$ be convex univalent in $U$ with $q(0)=1, q(z) \neq 0, z \in U$, and assume that $q$ satisfies (3.5). Suppose that $\frac{z q^{\prime}(z)}{q(z)}$ is starlike univalent in $U$. Let $f \in A$ satisfies

$$
\frac{z^{1-\delta} D_{z}^{\delta} I_{\lambda_{1}, \lambda_{2}, \ell, d}^{n, m+1} f(z)}{\Gamma(2-\delta)\left(D_{z}^{\delta} I_{\lambda_{1}, \lambda_{2}, \ell, d}^{n, m} f(z)\right)^{2}} \in H[q(0), 1] \cap Q
$$

and $\Omega\left(\eta_{1}, \eta_{2}, \eta_{3}, \eta_{4}, t, \delta, n, m, \lambda_{1}, \lambda_{2}, \ell, d ; z\right) \quad$ is univalent in $U$, where $\Omega\left(\eta_{1}, \eta_{2}, \eta_{3}, \eta_{4}, t, \delta, n, m, \lambda_{1}, \lambda_{2}, \ell, d ; z\right)$ is given by $(2.11)$. If

$$
\eta_{1}+\eta_{2} q(z)+\eta_{3} q^{2}(z)+\eta_{4} q^{3}(z)+t \frac{z q^{\prime}(z)}{q(z)} \prec \Omega\left(\eta_{1}, \eta_{2}, \eta_{3}, \eta_{4}, t, \delta, n, m, \lambda_{1}, \lambda_{2}, \ell, d ; z\right),
$$

then

$$
q(z) \prec \frac{z^{1-\delta} D_{z}^{\delta} I_{\lambda_{1}, \lambda_{2}, \ell, d}^{n, m+1} f(z)}{\Gamma(2-\delta)\left(D_{z}^{\delta} I_{\lambda_{1}, \lambda_{2}, \ell, d}^{n, m} f(z)\right)^{2}}
$$

and $q$ is the best subordinant of (3.9).

Proof. Define the function $p$ by

$$
p(z)=\frac{z^{1-\delta} D_{z}^{\delta} I_{\lambda_{1}, \lambda_{2}, \ell, d}^{n, m+1} f(z)}{\Gamma(2-\delta)\left(D_{z}^{\delta} I_{\lambda_{1}, \lambda_{2}, \ell, d}^{n, m} f(z)\right)^{2}}, \quad z \in U .
$$

Then the function $p$ is analytic in $U$ and $p(0)=1$.

We note that

$$
\Omega\left(\eta_{1}, \eta_{2}, \eta_{3}, \eta_{4}, t, \delta, n, m, \lambda_{1}, \lambda_{2}, \ell, d ; z\right)=\eta_{1}+\eta_{2} p(z)+\eta_{3} p^{2}(z)+\eta_{4} p^{3}(z)+t \frac{z p^{\prime}(z)}{p(z)},
$$

where $\Omega\left(\eta_{1}, \eta_{2}, \eta_{3}, \eta_{4}, t, \delta, n, m, \lambda_{1}, \lambda_{2}, \ell, d ; z\right)$ is given by (2.11). From (3.9) and (3.11), we obtain

$$
\eta_{1}+\eta_{2} q(z)+\eta_{3} q^{2}(z)+\eta_{4} q^{3}(z)+t \frac{z q^{\prime}(z)}{q(z)} \prec \eta_{1}+\eta_{2} p(z)+\eta_{3} p^{2}(z)+\eta_{4} p^{3}(z)+t \frac{z p^{\prime}(z)}{p(z)} .
$$

The remaining part of the proof Theorem 3.3 is similar to that of Theorem 3.2 and hence we omit it. 


\section{SANDWICH Results}

Combining results of differential subordinations and superordinations, we state the following "sandwich results".

Theorem 4.1. Let $q_{1}$ and $q_{2}$ be convex univalent in $U$ with $q_{1}(0)=q_{2}(0)=1$. Suppose $q_{2}$ satisfies (2.1), $\gamma>0$ and $\operatorname{Re}\{\sigma\}>0$. Let $f \in A$ satisfies

$$
\left(\frac{\Gamma(2-\delta) D_{z}^{\delta} I_{\lambda_{1}, \lambda_{2}, \ell, d}^{n, m} f(z)}{z^{1-\delta}}\right)^{\gamma} \in H[1,1] \cap Q
$$

and

$$
\begin{aligned}
& \left(1-\frac{\sigma\left[\ell\left(1+\left(\lambda_{2}(k-1)\right)+d\right]\right.}{\ell \lambda_{1}(1-\delta)}\right)\left(\frac{\Gamma(2-\delta) D_{z}^{\delta} I_{\lambda_{1}, \lambda_{2}, \ell, d}^{n, m} f(z)}{z^{1-\delta}}\right)^{\gamma} \\
& +\frac{\sigma\left[\ell\left(1+\left(\lambda_{2}(k-1)\right)+d\right]\right.}{\ell \lambda_{1}(1-\delta)}\left(\frac{\Gamma(2-\delta) D_{z}^{\delta} I_{\lambda_{1}, \lambda_{2}, \ell, d}^{n, m} f(z)}{z^{1-\delta}}\right)^{\gamma}\left(\frac{D_{z}^{\delta} I_{\lambda_{1}, \lambda_{2}, \ell, d}^{n, m+1} f(z)}{D_{z}^{\delta} I_{\lambda_{1}, \lambda_{2}, \ell, d}^{n,} f(z)}\right)
\end{aligned}
$$

be univalent in $U$. If

$$
\begin{aligned}
& q_{1}(z)+\frac{\sigma}{(1-\delta) \gamma} z q_{1}^{\prime}(z) \\
\prec & \left(1-\frac{\sigma\left[\ell\left(1+\left(\lambda_{2}(k-1)\right)+d\right]\right.}{\ell \lambda_{1}(1-\delta)}\right)\left(\frac{\Gamma(2-\delta) D_{z}^{\delta} I_{\lambda_{1}, \lambda_{2}, \ell, d}^{n, m} f(z)}{z^{1-\delta}}\right)^{\gamma} \\
& +\frac{\sigma\left[\ell\left(1+\left(\lambda_{2}(k-1)\right)+d\right]\right.}{\ell \lambda_{1}(1-\delta)}\left(\frac{\Gamma(2-\delta) D_{z}^{\delta} I_{\lambda_{1}, \lambda_{2}, \ell, d}^{n, m} f(z)}{z^{1-\delta}}\right)^{\gamma}\left(\frac{D_{z}^{\delta} I_{\lambda_{1}, \lambda_{2}, \ell, d}^{n, m+1} f(z)}{D_{z}^{\delta} I_{\lambda_{1}, \lambda_{2}, \ell, d}^{n,} f(z)}\right) \\
\prec & q_{2}(z)+\frac{\sigma}{(1-\delta) \gamma} z q_{2}^{\prime}(z),
\end{aligned}
$$

then

$$
q_{1}(z) \prec\left(\frac{\Gamma(2-\delta) D_{z}^{\delta} I_{\lambda_{1}, \lambda_{2}, \ell, d}^{n, m} f(z)}{z^{1-\delta}}\right)^{\gamma} \prec q_{2}(z)
$$

and $q_{1}$ and $q_{2}$ are, respectively, the best subordinant and the best dominant.

Theorem 4.2. Let $q_{1}$ and $q_{2}$ be convex univalent in $U$ with $q_{1}(0)=q_{2}(0)=1$. Suppose $q_{1}$ satisfies (3.5) and $q_{2}$ satisfies (2.5). Let $f \in A$ satisfies

$$
\left(\frac{D_{z}^{\delta} I_{\lambda_{1}, \lambda_{2}, \ell, d}^{n, m+1} f(z)}{D_{z}^{\delta} I_{\lambda_{1}, \lambda_{2}, \ell, d}^{n, m} f(z)}\right)^{\gamma} \in H[1,1] \cap Q
$$


and $\Psi\left(\eta_{1}, \eta_{2}, \eta_{3}, \eta_{4}, \gamma, t, \delta, n, m, \lambda_{1}, \lambda_{2}, \ell, d ; z\right) \quad$ is univalent in $U$, where $\Psi\left(\eta_{1}, \eta_{2}, \eta_{3}, \eta_{4}, \gamma, t, \delta, n, m, \lambda_{1}, \lambda_{2}, \ell, d ; z\right)$ is given by $(2.7)$. If

$$
\begin{aligned}
\eta_{1}+\eta_{2} q_{1}(z)+\eta_{3} q_{1}^{2}(z)+\eta_{4} q_{1}^{3}(z)+t \frac{z q_{1}^{\prime}(z)}{q_{1}(z)} & \prec \Psi\left(\eta_{1}, \eta_{2}, \eta_{3}, \eta_{4}, \gamma, t, \delta, n, m, \lambda_{1}, \lambda_{2}, \ell, d ; z\right) \\
& \prec \eta_{1}+\eta_{2} q_{2}(z)+\eta_{3} q_{2}^{2}(z)+\eta_{4} q_{2}^{3}(z) \\
& +t \frac{z q_{2}^{\prime}(z)}{q_{2}(z)},
\end{aligned}
$$

then

$$
q_{1}(z) \prec\left(\frac{D_{z}^{\delta} I_{\lambda_{1}, \lambda_{2}, \ell, d}^{n, m+1} f(z)}{D_{z}^{\delta} I_{\lambda_{1}, \lambda_{2}, \ell, d}^{n, m} f(z)}\right)^{\gamma} \prec q_{2}(z)
$$

and $q_{1}$ and $q_{2}$ are, respectively, the best subordinant and the best dominant.

Theorem 4.3. Let $q_{1}$ and $q_{2}$ be convex univalent in $U$ with $q_{1}(0)=q_{2}(0)=1$. Suppose $q_{1}$ satisfies (3.5) and $q_{2}$ satisfies (2.5). Let $f \in A$ satisfies

$$
\frac{z^{1-\delta} D_{z}^{\delta} I_{\lambda_{1}, \lambda_{2}, \ell, d}^{n, m+1} f(z)}{\Gamma(2-\delta)\left(D_{z}^{\delta} I_{\lambda_{1}, \lambda_{2}, \ell, d}^{n, m} f(z)\right)^{2}} \in H[1,1] \cap Q
$$

and $\Omega\left(\eta_{1}, \eta_{2}, \eta_{3}, \eta_{4}, t, \delta, n, m, \lambda_{1}, \lambda_{2}, \ell, d ; z\right) \quad$ is univalent in $U$, where $\Omega\left(\eta_{1}, \eta_{2}, \eta_{3}, \eta_{4}, t, \delta, n, m, \lambda_{1}, \lambda_{2}, \ell, d ; z\right)$ is given by (2.11). If

$$
\begin{aligned}
\eta_{1}+\eta_{2} q_{1}(z)+\eta_{3} q_{1}^{2}(z)+\eta_{4} q_{1}^{3}(z)+t \frac{z q_{1}^{\prime}(z)}{q_{1}(z)} & \prec \Omega\left(\eta_{1}, \eta_{2}, \eta_{3}, \eta_{4}, t, \delta, n, m, \lambda_{1}, \lambda_{2}, \ell, d ; z\right) \\
& \prec \eta_{1}+\eta_{2} q_{2}(z)+\eta_{3} q_{2}^{2}(z)+\eta_{4} q_{2}^{3}(z) \\
& +t \frac{z q_{2}^{\prime}(z)}{q_{2}(z)},
\end{aligned}
$$

then

$$
q_{1}(z) \prec \frac{z^{1-\delta} D_{z}^{\delta} I_{\lambda_{1}, \lambda_{2}, \ell, d}^{n, m+1} f(z)}{\Gamma(2-\delta)\left(D_{z}^{\delta} I_{\lambda_{1}, \lambda_{2}, \ell, d}^{n, m} f(z)\right)^{2}} \prec q_{2}(z)
$$

and $q_{1}$ and $q_{2}$ are, respectively, the best subordinant and the best dominant.

Remark 4.1. By specifying the function $\phi$ and selecting the particular values of $\eta_{1}, \eta_{2}, \eta_{3}, \eta_{4}, \gamma, \delta, n, m, \lambda_{1}, \lambda_{2}, \ell$ and $d$, we can derive a number of known results. Some of them are given below.

(1) Taking $\delta=n=\lambda_{2}=d=0$ and $\ell=1$ in Theorems 2.1, 3.1, 4.1, we get the results obtained by Răducanu and Nechita [10, Theorem 3.1, Theorem 3.6, Theorem 3.9].

(2) Putting $\delta=n=\lambda_{2}=\eta_{1}=\eta_{3}=\eta_{4}=d=0, \eta_{2}=\ell=1$ and $\phi(w)=t$ in Theorems $2.3,3.3,4.3$, we obtain the results obtained by Nechita $[8$, Theorem 14, Theorem 19, Corollary 21]. 
(3) For $\delta=n=\lambda_{2}=\eta_{1}=\eta_{3}=\eta_{4}=d=0, \lambda_{1}=\eta_{2}=\ell=1$ and $\phi(w)=t$ in Theorems $2.3,3.3,4.3$, we have the results obtained by Shanmugam et al. [13, Theorem 5.4, Theorem 5.5, Theorem 5.6].

(4) By taking $\delta=n=m=\lambda_{2}=\eta_{1}=\eta_{3}=\eta_{4}=d=0, \lambda_{1}=\eta_{2}=\ell=1$ and $\phi(w)=t$ in Theorems $2.3,3.3,4.3$, we get the results obtained by Shanmugam et al. [13, Theorem 3.4, Theorem 3.5, Theorem 3.6].

(5) Putting $\delta=n=\lambda_{2}=\eta_{1}=\eta_{3}=\eta_{4}=0, \eta_{2}=\ell=1$ and $\phi(w)=t$ in Theorems $2.3,3.3,4.3$, we have the results obtained by Shammaky [12, Theorem 3.4, Theorem 3.5, Theorem 3.6].

(6) Taking $\delta=n=m=\lambda_{2}=d=0$ and $\lambda_{1}=\ell=1$ in Theorem 2.1, we obtain the results obtained by Murugusundaramoorthy and Magesh [7, Corollary 3.3].

(7) Putting $\delta=n=m=\lambda_{2}=d=0$ and $\lambda_{1}=\ell=1$ in Theorems 3.1, 4.1, we obtain the results obtained by Răducanu and Nechita [10, Corollary 3.7, Corollary 3.10].

Acknowledgements. The authors would like to thank the referee(s) for their helpful comments and suggestions.

\section{REFERENCES}

[1] R. M. Ali, V. Ravichandran, M. H. Khan and K. G. Subramanian, Differential sandwich theorems for certain analytic functions, Far East Journal of Mathematical Sciences 15(1) (2004), 87-94.

[2] A. A. Attiya and M. F. Yassen, Some subordination and superordination results associated with generalized Srivastava-Attiya operator, Filomat 31(1) (2017), 53-60.

[3] T. Bulboacǎ, Classes of first order differential superordinations, Demonstr. Math. 35(2) (2002), 287-292.

[4] S. P. Goyal, P. Goswami and H. Silverman, Subordination and superordination results for a class of analytic multivalent functions, Journal of Inequalities in Pure and Applied Mathematics (2008), Article ID 561638, 1-12.

[5] S. S. Miller and P. T. Mocanu, Differential Subordinations: Theory and Applications, Series on Monographs and Textbooks in Pure and Applied Mathematics 225, Marcel Dekker Inc., New York, Basel, 2000.

[6] S. S. Miller and P. T. Mocanu, Subordinants of differential superordinations, Complex Variables 48(10) (2003), 815-826.

[7] G. Murugusundaramoorthy and N. Magesh, Differential subordinations and superordinations for analytic functions defined by Dziok-Srivastava linear operator, J. Ineq. Pure Appl. Math. 7(4) (2006), 1-20.

[8] V. O. Nechita, Differential subordinations and superordinations for analytic functions defined by the generalized Salagean derivative operator, Acta Univ. Apulensis Math. Inform. 16 (2008), $14-156$.

[9] A. Oshah and M Darus, Differential sandwich theorems with new generalized derivative operator, Adv. Math. Sci. 3(2) (2014), 117-125.

[10] D. Răducanu and V. O. Nechita, A differential sandwich theorem for analytic functions defined by the generalized Salagean operator, Aust. J. Math. Anal. Appl. 9(1) (2012), 1-7.

[11] St. Ruscheweyh, New certain for univalent functions, Proc. Amer. Math. Soc. 49 (1975), 109 115 . 
[12] A. Shammaky, Differential sandwich theorems for analytic functions defined by an extended multiplier transformation, Advances in Pure Mathematics 2 (2012), 323-329.

[13] T. N. Shanmugam, V. Ravichandran and S. Sivasubramanian, Differential sandwich theorems for some subclasses of analytic functions, Aust. J. Math. Anal. Appl. 3(1) (2006), 1-11.

[14] T. N. Shanmugam, S. Sivasubramanian and H. Silverman, On sandwich theorems for some classes of analytic functions, Int. J. Math. Math. Sci. (2006), Article ID 29684, 1-13.

[15] H. M. Srivastava and S. Owa (Eds.), Current Topics in Analytic Function Theory, World Scientific Publishing Company, Singapore, 1992.

[16] N. Tuneski, On certain sufficient conditions for starlikeness, Int. J. Math. Math. Sci. 23(8) (2000), 521-527.

[17] A. K. Wanas, Differential sandwich theorems for integral operator of certain analytic functions, General Mathematics Notes 15(1) (2013), 72-83.

[18] A. K. Wanas, On sandwich theorems for higher-order derivatives of multivalent analytic functions associated with the generalized Noor integral operator, Asian-Eur. J. Math. 8(1) (2015), 1-14.

${ }^{1}$ Department of Mathematics, College of Science,

UNIVERSITY OF AL-QADISIYAH,

DIWANIYA, IRAQ

Email address: abbas.kareem.w@qu.edu.iq

${ }^{2}$ School of Mathematical Sciences,

Faculty of Science and Technology,

Universiti KebANGSAAN MALAYsia,

Bangi 43600,Selangor D. Ehsan, Malaysia

Email address: maslina@ukm.edu.my 